\title{
Electronic System for Orientation Control and Obstacle Avoidance for Underwater Glider without a Rudder
}

\author{
Yuvraj N. Patil ${ }^{1}$, V. S. Aneesh ${ }^{2}$, M. T. Abhilash ${ }^{3}$, and Swati Kadlag ${ }^{4}$ \\ ${ }^{1}$ Student, Dept. of Electronics and Telecommunication Engineering, \\ Symbiosis Institute of Technology (a constituent of SIU), \\ Pune - 412115, India. \\ yuvraj.n.patil22@gmail.com \\ ${ }^{2}$ Technical officer, Defence Avionics Research Establishment (DARE), \\ DRDO-Bangalore, India. \\ aneeessh@rediffmail.com \\ ${ }^{3}$ Prof., Dept. of Electronics, DRDO-DIAT(DU), \\ Pune, India. \\ chipmaker2005@gmail.com \\ ${ }^{4}$ Prof., Dept. of Electronics, Symbiosis Institute of Technology (a constituent of SIU), \\ Pune - 412115, India. \\ swatik@sitpune.edu.in
}

\begin{abstract}
Autonomous underwater gliders are classified in buoyancy operated long endurance underwater vehicles. The glide control algorithm has been developing for many designs. A system is proposed as an improvement to the existing designs to achieve glide control with 3 chamber internal hydraulic system and to avoid obstacles in its saw tooth motion profile and the orientation control that can be achieved with injection of fluids in internal tanks instead of relying on rudder or movable internal mass. Two algorithms for obstacle avoidance and correcting its approach vectors i.e. a self correcting mechanism with sensors and servo motors is represented. The onboard embedded system developed with payload sensors, navigation, communication and actuator control of the prototype design is also discussed in general.
\end{abstract}

Keywords: Gyroscope; Ultrasonic; compass; Obstacle; Servo; Prototype; Compensation; Orientation.

\section{Introduction}

An autonomous underwater glider goes in a saw tooth motion with some changes in its buoyancy. Such vehicles are small, low power and long endurance and are used for many applications [1]. The hydrodynamics of existing underwater gliders have been deployed and tested in various environments [2]. However there can be more techniques than those used for controlling the motion of a glider. There exists a scope for smart techniques like obstacle avoidance, change in use of rudders and movable internal mass to control the steering of the glider etc. 
In this paper, we are proposing a new approach to achieve a better propagation of the glider with help of orientation sensors. The results obtained by the existing gliders show a random motion of the glider into the ocean and collection of the data at those points. The ocean can pose various unwanted disturbances and obstacles. The gliders are likely to be deployed in coastal regions or low depth regions where obstacles like coral reefs, underwater mountains and marine life can cause threat to the glider [3]. To avoid this, glider must be able to detect the obstacle and avoid it by modifying its path of propagation. For this purpose, sensors like ultrasonic sensors, gyroscopes and accelerometers are integrated with system. The steering of the glider would become more difficult and complex if the rudder encounters malfunctions or if there is no rudder at all. For such situation, there's an alternative system for steering with movable internal mass mechanism. This system can be altered by utilizing the water around the glider to change its orientation. By letting in a controlled amount of water in chambers can provide necessary tilt and the vehicle can glide in that direction as a result of upward buoyant force and the resultant angle of the wings.

A system on chip approach is selected as the main control unit of the platform because of its flexibility of design, higher performance in terms of speed, computing power, better resource management than conventional microcontrollers, low cost and low power implementations. The current design uses PSoC5LP platform for the development of the control system. Obstacle avoidance and orientation control algorithms have been developed to adapt to the changes in environmental conditions of the glider. Some of the orientation sensors and navigation system have been interfaced according to the needs of the project. The glider by NASLab of Michigan Technical university [14] is based on single buoyancy tank. We are proposing a 3 cylinder approach. A detailed description of the interface and algorithm has been specified for the prototype design.

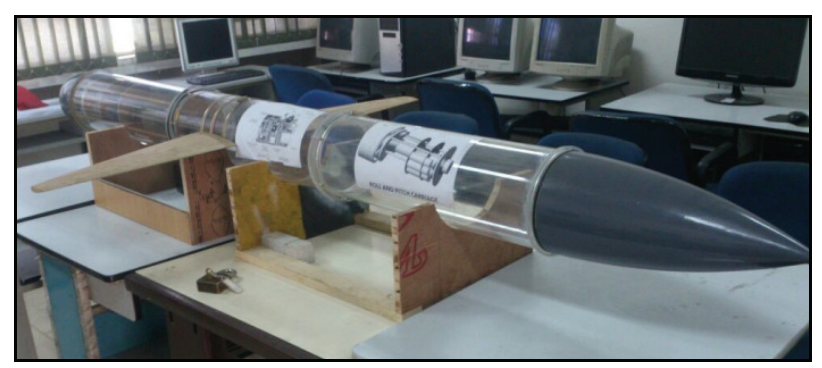

Fig. 1. Prototype of Underwater Glider designed by: Naval Constructors' Wing (NCW), IIT Delhi.

\section{Previous Work}

Initial models of some of the underwater gliders were Slocum battery and thermal, spray, sea glider etc. All these gliders use different methods to vary the buoyancy for its gliding motion in a saw tooth pattern. Each dive cycle consists of diving to a certain depth and rising to the surface. Almost all existing gliders depend on hydraulic type buoyancy engines powered by batteries. The Slocum thermal is an exception to these gliders and relies on 
thermalocline of ocean to convert solid medium to liquid and again from liquid to solid state [4]. It results in its displacement and helps to propagate in desired direction. However, this method restricts its functionality to areas where thermocline is well developed.

The Seaglider has a TT8 microcontroller as its electronic platform which uses Motorola MC68332 M.C. with 12-bit ADC and power management circuits [5]. It basically has conductivity temperature depth (CTC) sensing components on board. The Seaglider relies on a satellite communication link to transmit onboard data to the mission computer [6]. Whereas the Spray is a TT8 controlled glider that utilizes subsystems to control attitude and buoyancy variations, data acquisition, storage and transmission over satellite channel [7]. Along with CTDs, the spray also has flurometers, altimeter, Iridium satellite communication system, turbidity and dissolved oxygen measurement system.

The author [8] described selection of piston operated tanks for the prototype. The basic algorithm of the buoyancy engine was clockwise and anti-clockwise motion of a DC motor achieved that simulated to and forth motion of the piston in tanks that would result in a saw tooth profile. The onboard sensors included a temperature and a pressure sensor. The data from these sensors was stored onto a SD card and transmitted using XBee s2b Pro wireless Zigbee module after each dive cycle. However, the system was neither mounted on glider body nor was tested in actual or virtual environments.

\section{Present Prototype Design}

The microcontroller platform for the onboard control system remains to be PSoC5LP from Cypress. The data storage is in SD card as done previously and the trans-receiver module is still XBee s2b Pro. However some modifications to the existing design have been made as follows.

\subsection{Buoyancy Engine}

In previous design, a DC motor simulated the action of a piston operated buoyancy engine. The design approach was for a single cylinder mount for the periodic charging and discharging to result into a saw tooth motion. However the motor did not drive the piston mechanism and just simulated the change in rotation periodically. Also the design did not take into account the steering or the orientation control system of the glider. This gave rise to following objectives for a modified design.

- Build a piston operated cylinder structure for testing the algorithms.

- Couple the DC motor to a rack and pinion mechanism to drive a piston of a syringe cylinder.

- Modify the engine to a 3 cylinder design to provide orientation control

- Interface servo motors to operate pistons precisely

Unlike the spray that had a movable internal mass mechanism for steering [9], the design would make use of water from outside the glider body for similar operation. This system is also suitable for scenarios where rudder is not present or has encountered a malfunction. 

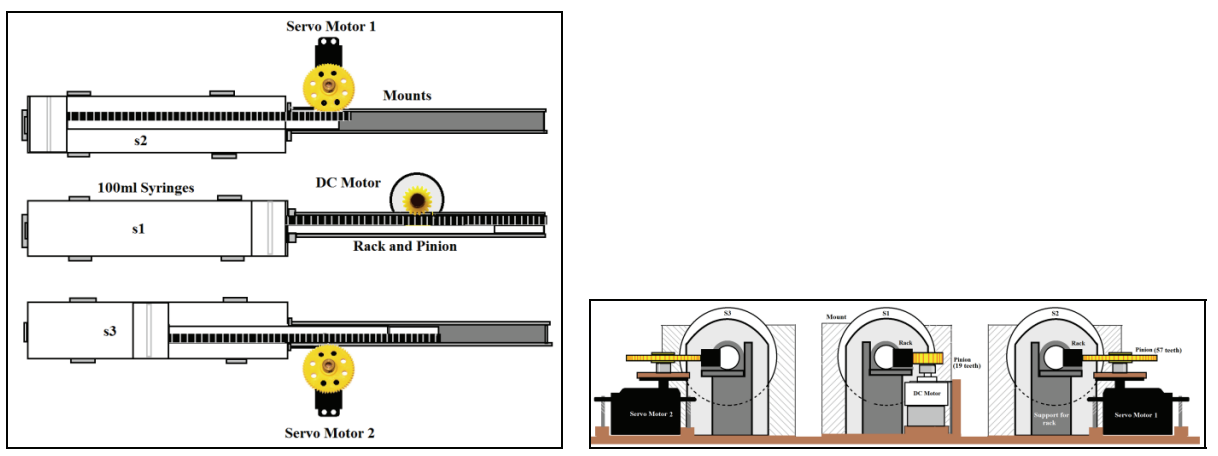

Fig. 2 \& 3. Top view of the design of demonstration set up,Front view of the design showing position of servo motors and a DC motor with respect to cylinders.
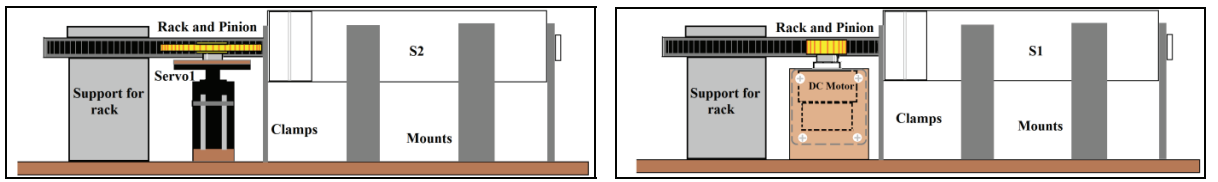

Fig. 4 \& 5. Side view of the servo controlled cylinder. Servo motor is connected to a rack and pinion assembly, Side view of the DC motor controlled cylinder responsible for saw tooth profile of the glider motion.
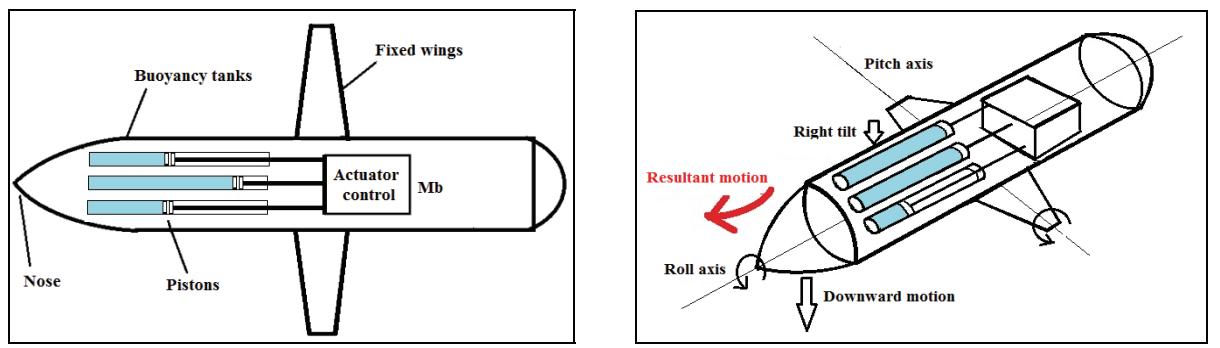

Fig. 6 \& 7. Buoyancy engine for the prototype design showing location of the cylinders inside the body of the glider, Glider turning right as one side tank becomes heavier by letting in more water than the other tank.

Figures 2, 3, 4 and 5 show the schematics of the proposed three tanks of the glider. However this placement is not tested on actual prototype glider body for the functionality neither is the position of those cylinders fixed as shown in above figures. The control system block consists of motors and rack and pinions that drive the pistons in those cylinders.

The position of the tanks inside the body is shown in Figure 6 and orientation change with respect to initial position is shown in Figure 7. The modeling parameters for the mechanical system are skipped in this paper and more emphasis is given on developing an algorithm for their operation. 


\subsection{Onboard Sensors and Drivers}

The board has now been interfaced with orientation sensors which include a 3 axis analog accelerometer (ADXL335), a digital gyroscope (MPU6050) and a digital compass (HMC5883L). The payload includes a BMP085 pressure and temperature sensor and a GPS module. Two HCSR04 ultrasonic sensors are interfaced for obstacle avoidance. The PSoC5 at the receiver end has been replaced by a XBee programmer module connected directly to PC via USB cable.

The gyroscope, BMP085, and digital compass have i2c interface with PSoC5LP where as the ultrasonic sensors are PWM operated. These sensors are basic robotics sensors and might not be suitable for underwater applications. These sensors have similar interfaces and are used to develop the algorithms. The paper includes making use of the data acquired from these sensors for developing the control algorithm. The DC motor is interfaced via LM369 motor driver IC as shown.

\subsection{Navigation System and Link with Base Station}

As the glider surfaces during its saw tooth evolution [1], the base station needs to acquire a fix on the current location of the glider. Based on important parameters such as high sensitivity of $165 \mathrm{dBm}, 22 \mathrm{~mA} 3.3 \mathrm{~V}$ low power consumption is made. UART interface, NMEA format Output, the GPS module SKG13C from SkyLab is selected for the application. Though the module is accurate up to 3 meters, it is cheap and user friendly. Also the module is low power and gives complete information data with position, velocity and time information in serial NMEA protocol. The module is suitable for most of the portable devices.

The module works on input voltage between $3.0 \mathrm{~V}$ to $4.2 \mathrm{~V}$ and on minimum current of $100 \mathrm{~mA}$. The receiver supports active antenna or passive antenna with maximum gain of $25 \mathrm{~dB}$. The module has on full duplex serial channel UART. This port is connected to the UART of PSoC5LP to receive data frames continuously that are being acquired form available satellites. However the acquisition is not continued. The data reception only takes place when the glider is on surface and the reception gets cut off when the glider goes inside the water. The GPS module requires $36 \mathrm{sec}$. for cold start and $1 \mathrm{sec}$. for hot start. This time depends upon the time for which the glider stays inside the water. The frames are in NMEA format and consist of GGA-Global Positioning System Fixed Data, GSA-GNSS DOP and Active Satellites, GSVGNSS Satellites in View and RMC-Recommended Minimum Specific GNSS Data required information which can be extracted and recorded.

For the communication with base station, XBee Pro $s 2 b$ trans-receiver is selected. The module operates in ISM band $(2.4 \mathrm{GHz})$ and has maximum data rate of $250 \mathrm{kbps}$. The Pro series modules have a longer range compared to other modules which is 2 miles of outdoor coverage in line of sight. And $200 \mathrm{~m}$ in indoor scenarios with transmission power of $63 \mathrm{~mW}$. The module is low power and operates in voltage range between $2.6 \mathrm{~V}$ to $3.3 \mathrm{~V}$.

\subsection{Embedded System}

The embedded system required for this application is supposed to be low power, low cost and versatile in nature. The low power operation of the microcontroller gives the 


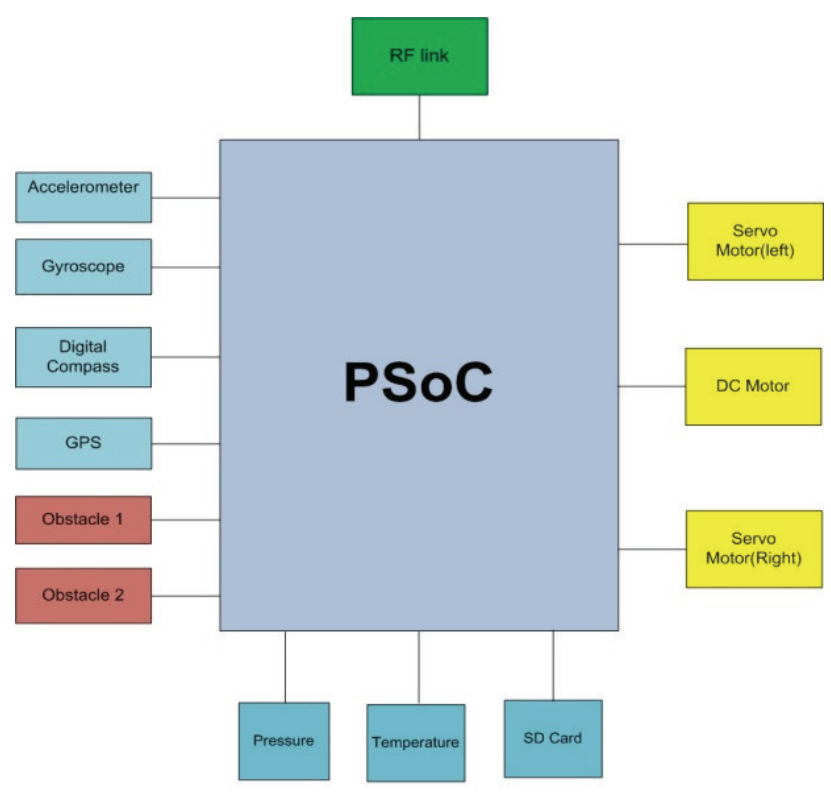

Fig. 8. Onboard interfaces with PSoC5LP development board.

glider its long endurance while its versatile nature supports various sensor and actuator interfaces which can vary in future as well [3]. Hence for this application a system on chip (SoC) approach is selected. SoCs are low power, Re-programmable, resource saving powerful computing devices [6]. PSoC5LP has 256KB flash memory, $64 \mathrm{~KB}$ of SRAM, and SD card interface for data storage accompanied by a 12-bit Delta sigma A/D converter and a 20-bit Successive Approximation Rate (SAR) A/D converter.

It has over 64 GPIOs (General Purpose Input Output) which can be configured as analog or digital input/outputs. It also supports I2C and SPI protocols, which are required by most digital output sensors. Figure 8 shows interfacing on transmitter side and receiver side.

On the receiver side of the system, the XBee module is mounted on a programmer board from Sparkfun electronics and is directly interfaced with mission computer via USB cable. The data reception on the PC is done with help of Realterm software [15] that captures the incoming data on serial ports.

\section{Obstacle Avoidance Algorithm}

\subsection{Ultrasonic Sensors}

Since sound has proved to travel farther in water without much fading than radio waves, sonar has been an effective technology in detecting objects at farther distances without an actual contact with main body. For interface purpose and developing an algorithm two HC-SR04 ultrasonic sensors are interfaced with PSoC5LP. The sensors can detect any solid obstacle up to 400 centimeters. The reason behind using two such 
sensors is that the glider should not consider a small fish or some junk sinking in the water as an obstacle. By comparing both sensors the PSoC5LP can determine whether or not the obstacle is large enough to change motion of the glider, these sensors con detect obstacle from 0 degrees to 15 degrees. So they can be placed on both wings or in the nose of the glider to cover required area.

\subsection{Interfacig}

Two HC-SR04 ultrasonic sensors are interfaced with PSoC5LP and placed at a distance of $3 \mathrm{~cm}$ from each other for demonstration purpose.

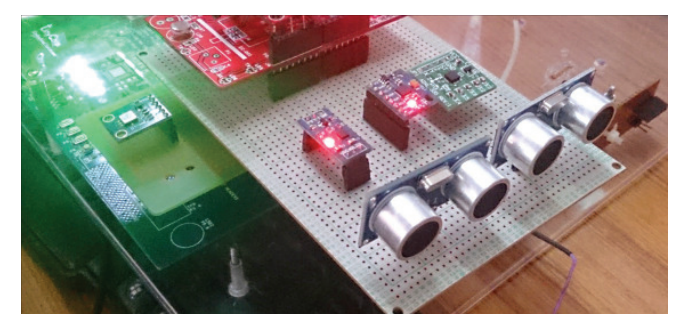

Fig. 9. Two ultrasonic sensors interfaced with PSoC5LP.

On the actual glider, they can be placed on both wings to cover more area and better obstacle detection. The sensors are PWM type and needs a trigger of 10 micro seconds after which they send out 8 pulses of $40 \mathrm{kHz}$ and then weight for an echo. A counter runs for that amount of time till the echo pulse is received. Both the sensors give almost same distance with offset of $1 \mathrm{~cm}$. The distance is calculated based on following equations.

$$
\text { Range }=\frac{\text { echotime } \times \text { velocity of sor }}{2}
$$

There will be some noise present in the received echo but can be easily dealt by applying certain threshold. Complex filters are not required since there purpose of these sensors is just to detect an obstacle and not to map an entire terrain.

The algorithm is for obstacle avoidance applied for the central buoyancy tank since this is the tank that would be responsible for the saw tooth motion of the glider. As the water is being drawn into the tank, the glider is going down. If the obstacle is detected, the piston motion is reversed so as to push the water out as a result the glider starts upward motion before reaching its predefined depth to avoid the obstacle. The AUG central piston and tank is imitated by a $100 \mathrm{ml}$ surgical syringe operated by rack and pinion mounted on a wooden platform. Since this is a prototype used for simulation purpose and developing an algorithm, the dimensions and design parameters that would suit actual glider are neglected at this moment. The displacement of the rack is given by following formula:

$$
x=2 \Pi r \Theta
$$


where,

$\mathrm{x}$ is the displacement of rack,

$r$ is the radius of pinion,

$\Theta$ is the angle of rotation of pinion.
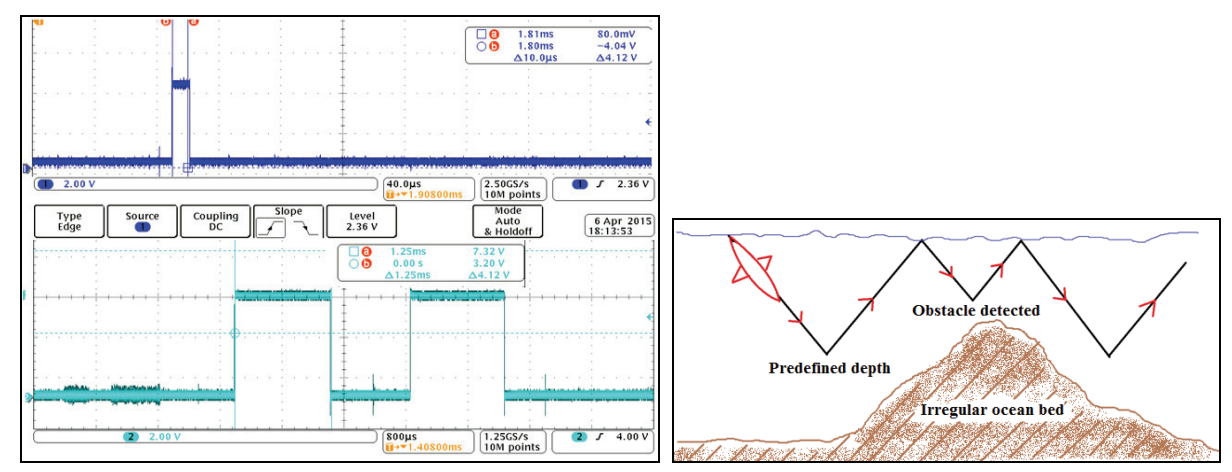

Fig. 10 \& 11. DSO showing echo received by sensor after triggering, Desired obstacle avoidance in saw toot motion.

The DC motor is turned clockwise for a duration of 5 seconds which is enough to pull the piston by full length of the cylinder. A short delay is of $2 \mathrm{sec}$. is introduced after which the motor goes in anti-clockwise direction. This pushes the piston back in its initial state depicting the surfacing action of the glider. The delay depends upon the counter being incremented per iterations which selected based on experimental observations.

A feedback is taken from ultrasonic sensors all the time to check for any obstacle. In case of an obstacle, the rotation of the motor is reversed.

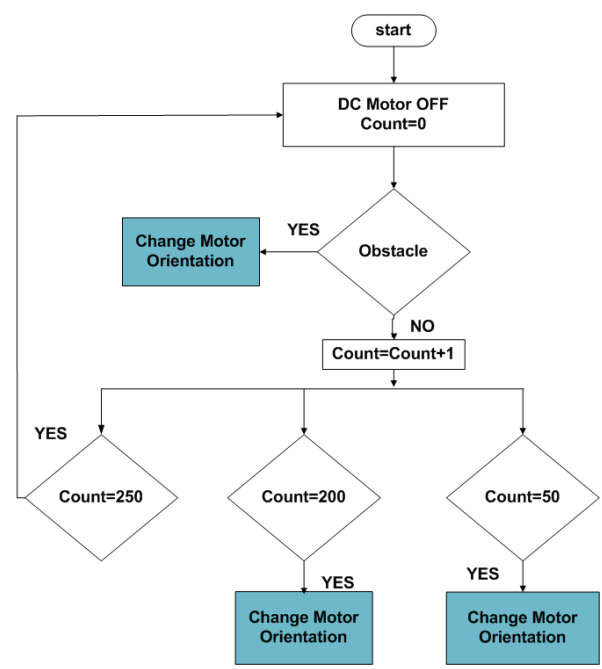

Fig. 12. Flowchart for obstacle avoidance system. 
This stops the glider from achieving predefined altitude and the glider will change its upward or downward motion. The glider does not steer itself in order to avoid obstacle hence the steering mechanism is independent of the obstacle avoidance system. Above is a flowchart of the algorithm for the obstacle avoidance system.

\section{Orientation Control Algorithm}

Many of the existing gliders that are designed rely on either rudder or movable internal mass to change its course. Assuming unavailability of the rudder on a glider and movable solid internal mass, a 3 cylinder mechanism is proposed to be used. The pistons of side cylinders are operated by two VS-2 servo motors. The motor shaft is connected to rack and pinion mechanisms that drive the pistons. The servo motors are PWM signal driven and their angle shift for respective compare values is shown in following diagram. The system clock for the PWM block in PSoC creator 3.0 is set at $2 \mathrm{MHz}$. and the period is kept 20 micro seconds. The angle will depend upon the compare value which is nothing but the duty cycle of PWM pulse. The pulse width is varied in order to change the duty cycle.
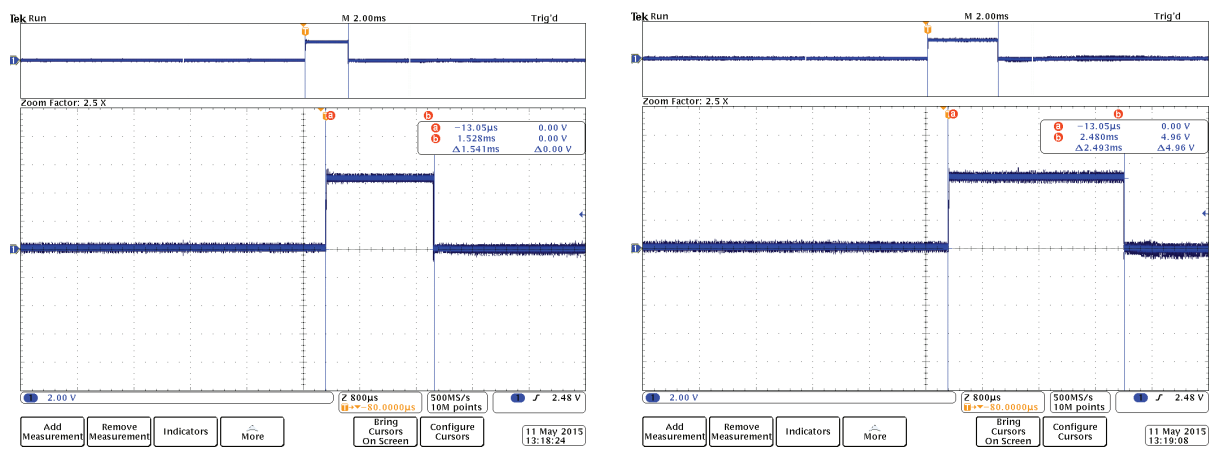

Fig. $13 \&$ 14. PWM for minimum angle of servo motor for piston at initial position (no water in tank), PWM for maximum angle of servo motor for piston at final position (water in tank).

At $1.53 \mathrm{~ms}$ of the pulse width, the angle of rotation of the servo motor is close to 0 degrees. On powering on the system, this is the default PWM pulse width given to the servo motor. At $2.40 \mathrm{~ms}$ of the pulse width the angle of rotation of the servo motor is close to +90 degrees. The on time for pulse tends to increase further on more deviation but would not result in servo motor's angle change beyond +90 degrees.

For understanding the concept behind the steering control, the angle of rotation of servo motor is kept directly proportional to the change in the difference of yaw angle from glider's initial approach vector. i.e. minimum the angle of deviation from main path lesser will the water allowed to fill up the tank and vice versa. This would result 


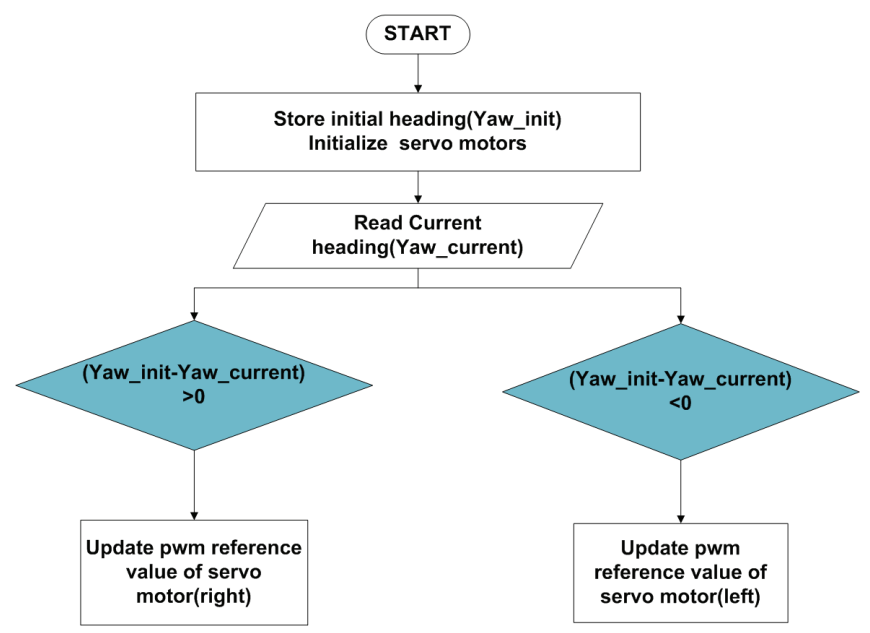

Fig. 15. Flowchart for orientation control using feedback from sensors.

in steering of the glider and compensating motion for unwanted shift in the course of the glider A feedback is taken from digital compass and the gyroscope. The compare value of PWM required to drive servo motor by -90 degrees is 600 and for +90 degrees is 2400 . The equations for mapping the difference in yaw angle with the compare value are.

$$
\begin{gathered}
\text { map }(\text { difference, } \min , \max , 600,2400) \\
\text { Compare value }=\left(\frac{(\text { difference in yaw }+90)}{180}\right) \times(2400-600)+600 \\
\text { Compare value }=[(\text { difference in yaw }+90)] 10+600
\end{gathered}
$$

This compare value is nothing but the pulse width that needs to be varied for the PWM signal to get desired angle on servo motor. The flowchart of the algorithm is shown in Figure 15.

The digital compass is a HMC5883L with i2c interface working on $2.6 \mathrm{~V}-3.6 \mathrm{~V}$. The sensitivity of this digital compass is 2 milli-gauss Field Resolution in \pm 8 Gauss Fields. As the digital compass may read any value while dropping the glider, depending upon the direction at that moment the reading is recorded and stored in a temporary variable. This variable is stored as a reference for further computing. The PSoC keeps reading its new heading direction and compares its current reading with this stored value. The current reading is subtracted from the stored value and the difference is noted. Depending upon positive or negative value of the difference, the deviation in left or right direction is determined. Same is done by using a digital gyroscope. The difference in the angle is calculated and compared with the digital compass readings.

The algorithm is implemented on piston mechanisms mounted on a wooden platform. Figure 18 shows actual demonstration board with cylinders mounted with rack and pinions driven by servo motors and DC motor. 


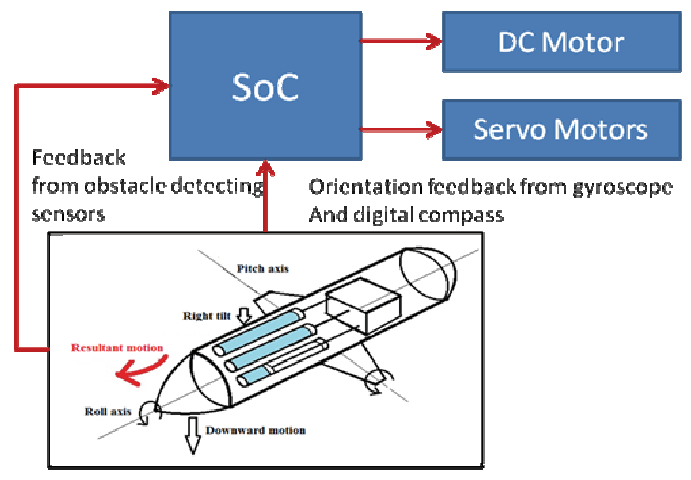

Fig. 16. Actuator control with feedback from sensors.

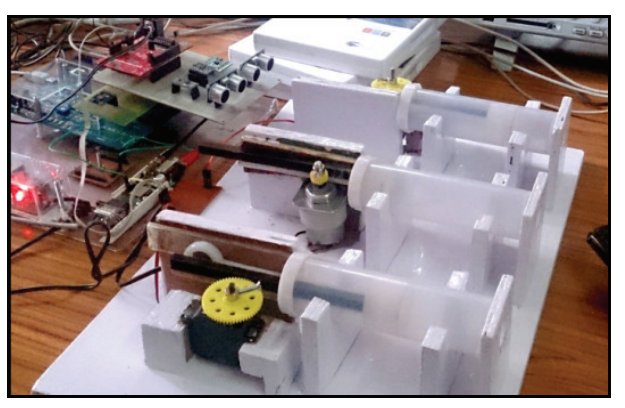

Fig. 17. Prototype set up of actuator control with PSoC5LP interface.

\section{Conclusion}

The orientation control and obstacle avoidance is achieved on a demonstration set up based on system of chip (SoC) platform with the help of sensors. The SoC approach provided better flexibility in programming and interfacing all the required sensors with minimum system resources. PSoC5LP powered by ARM-Cortex M3 has better performance than PSoC3 in terms of CPU and RAM utilization for a given program. This remains an initial stage of development of the prototype that is intended. The actuator selection for implementation is conceptual and intended to develop an algorithm. Also the mechanical parameters needed for selection are neglected at this stage. Currently the piston motion obtained by servo and DC motor based on sensor inputs is satisfactory. However the tuning of this system is significantly crude and needs precision.

The obstacle detection carried out by the ultrasonic sensors is based on a threshold set for a minimum range of $20 \mathrm{~cm}$. which can be changed based on the region of operation, velocity, length and weight of the glider. The intension of this system remains to detect the obstacle and not to map the terrain but can be considered for better accuracy. Similar algorithm can be implemented for underwater acoustic sensors as well. The motion of the piston in central cylinder is reversed by DC motor on an obstacle being placed before the sensors to simulate change in saw tooth profile. 
For orientation control, the min. and max. angles of servo motor drive the piston by its full length inside the cylinders. The difference in angles of heading vectors obtained from the orientation sensors is linearly proportional to the angle of rotation of both servo motors.

\section{Acknowledgement}

We would like to take this opportunity to express our heartiest gratitude to DIATDRDO(DU) Pune, and to all its students and faculty members of Electronics Engineering Department, Naval Wing, Navigation department, aerospace dpt. of DIATDRDO(DU) for their valuable suggestions and support throughout this work. We also thank Symbiosis Institute of Technology, Pune for providing valuable guidance and necessary facilities throughout the process.

\section{References}

1. Amit Ray, Singh S.N. and Seshadri V, 'Underwater Gliders - Force Multipliers for Naval Roles' at the RINA conference 'Warship 2011 : Naval Submarines and UUVs', 29-30 June, 2011 at the Guildhall, Bath, UK. William P Barker, 'Analysis of undersea glider architectures and an assessment of undersea glider integration for undersea applications', Sep 12.

2. Danio Joe, R V Shashank Shankar, RVijay kumar and Arnab Das, 'Concept Design of Autonomous Underwater Glider.

3. Arnab Das, "Naval Operations Analysis in the Indian Ocean Region-A Review", Accepted for publication in the IDSA Journal.

4. Russ E. Davis, Charles C.Eriksen, Clayton P. Jones. "Autonomous Buoyancy-driven Underwater gliders".The technology and application of autonomous underwater vehicles, G.Griffiths,ed., Taylor and Fransis, London 2002.

5. Charles C. Eriksen, T. James Osse, Russell D. Light, Timothy Wen, Thomas W. Lehman, Peter L. Sabin, John W. Ballard, and Andrew M. Chiodi Seaglider: A Long-Range Autonomous Underwater Vehicle for Oceanographic Research, IEEE Journal of oceanic Engineering, Vol. 26, No. 4, Oct 2001.

6. Stephen Bozzone, Brian Newberry, Dominic Germana Enhancements and Performance of Seaglider Iridium Communications System Chris Yahnker,OCEANS,2012.

7. Abdallah Ismail, Project report on Programmable System-on-Chip technology from Cypress Semiconductor digital systems architecture, Dec 2007.

8. M.D. Nair, Abhilash M.T., Vijay Kumar, Arnab Das "Embedded control system for underwater glider based on PSoC" et al International Journal of Computer and Electronics Research, Volume 3, Issue 2, April 2014

9. Jeff Sherman, Russ E. Davis, W. B. Owens, and J. Valdes, "The Autonomous Underwater Glider Spray” IEEE JOURNAL OF OCEANIC ENGINEERING, VOL. 26, NO. 4, OCTOBER 2001

10. Luke Stutters, Honghai Liu Senior Member, IEEE, Carl Tiltman and David J Brown,"Navigation Technologies for Autonomous Underwater Vehicles",IEEE TRANSACTIONS ON SYSTEMS, MAN AND CYBERNETICS - PART C: APPLICATIONS AND REVIEWS, VOL. 38, NO. 4, JULY 2008.

11. R. E. Davis, "Preliminary results from directly measuring middepth circulation in the tropical and South Pacific," J. Geophys. Res., vol. 103, pp. 24 619-24 639, 1998. 
Electronic System for Orientation Control and Obstacle Avoidance for Underwater Glider ...

12. M. J. McPhaden, "Genesis and evolution of the 1997-98 El Nino," Science, vol. 283, pp. 950-954, 1999.

13. S. A. Jenkins and J. Wasyl, "Optimization of glides for constant wind fields and course headings,” J. Aircraft, vol. 27, pp. 632-638, 1990.

14. http://www.mtu.edu/engineering/

15. http://realterm.sourceforge.net/ 